\title{
Remarks on the Load Dependency of the Voltage Quality of a 500MVA Synchronous Generator with Fractional Slot Windings
}

\author{
C. Grabner \\ Institute of Electrical M achines and D rives \\ Graz University of Technology \\ Kopernikusgasse $24, \mathrm{~A}-8010 \mathrm{Graz}$ (A ustria) \\ phone: +43 316873 8602, fax:+43 316873 8103, e-mail: grabner@ema.tu-graz.ac.at
}

\begin{abstract}
The induced voltage shape of an electrical generator is mainly depending on the used materials, the electromagnetic design and the actual operational state. Especially large synchronous hydro generators are often equipped with a fractional slot stator winding in order to fulfill the high requirements of a sinusoidal voltage output. However, due to such a stator winding, many different harmonic field wave components are circulating along the airgap. Additionally to the fundamental harmonic wave and the higher harmonics with wavelengths shorter than two pole pitches, the armature reaction of a fractional slot winding creates harmonic field components with a wavelength larger than two pole pitches, the so called subharmonics. The resulting voltage quality of a 500M V A salient pole synchronous generator is investigated with finite element methods separately for higher harmonics and sub-harmonics in dependence of various operational states.
\end{abstract}

\section{Key words}

Synchronous generator, V oltage shape quality, Fractional slot winding, Higher harmonics, Sub-harmonics.

\section{Introduction}

The most wanted high power quality is nowadays one of the main guidelines within the construction process of large synchronous generators. This aim can also be explained by the condition of vanishing higher harmonics in the induced stator voltage spectrum. Thus, a very detailed knowledge about the intensities of influences to the local distribution of the radial magnetic flux density inside the airgap is of essential interest for an optimized design process of the machine described in Table I.

Unfortunately, the basic view of the problem by applying the usually known fundamental wave theory is insufficient for such kind of investigations, which should also take account of different effects, such as e.g. nonlinearities and higher harmonics.

\section{TAB LE I. - Characteristics of the synchronous machine.}

\begin{tabular}{lccc}
\hline Rated apparent power & $\mathrm{S}_{\mathrm{N}}$ & 465 & $\mathrm{MVA}$ \\
Rated electrical stator current & $\mathrm{I}_{\mathrm{s}, \mathrm{N}}$ & 12784 & $\mathrm{~A}$ \\
Rated rotor excitation current & $\mathrm{I}_{\mathrm{f}, \mathrm{N}}$ & 2865 & $\mathrm{~A}$ \\
Rated electrical stator voltage & $\mathrm{U}_{\mathrm{N}}$ & 21000 & $\mathrm{~V} \mathrm{Y}$ \\
Rated frequency & $\mathrm{f}_{\mathrm{N}}$ & 50 & $\mathrm{~Hz}$ \\
Number of poles & $2 \mathrm{p}$ & 14 & \\
Number of stator phases & $\mathrm{m}$ & 3 & \\
Number of stator slots & $\mathrm{N}$ & 138 & \\
\hline
\end{tabular}

Within the past years, a more accurate and extended information about higher harmonics in the distribution of the radial component of the magnetic flux density along the airgap has been successfully developed with the aid of numerical methods [1]. Thereby, the main problem within the machine design seems to be the very high magnetic utilization of some active parts. Thus, the knowledge about the occurring nonlinear material influences of the iron parts become more crucial within an improved construction process [2,3].

First, there must be distinguished between the magneto motive force excitation even due to the rotor or the stator currents and the evoked magnetic flux distribution along the circumferential direction inside the airgap of the machine.

The Fourier series expansion of the stator current magneto motive force yields to harmonic wave magnitudes as

$$
V_{v} \sim V_{f} \frac{\xi_{v}}{v}
$$

where $V_{f}$ denotes the fundamental one. The magnitudes $V_{v}$ are proportional to the reciprocal value of the harmonic order $v$. The introduced stator winding factor

$$
\xi_{v}=\xi_{d, v} \xi_{p, v}
$$

is separated into distribution and pitch factors. Thereby, the distribution factor is given by

$$
\xi_{d, v}=\frac{\sin \left(v \frac{\pi}{2 m}\right)}{n q \sin \left(v \frac{\pi}{2 m n q}\right)},
$$

and the pitch factor is introduced as

$$
\xi_{p, v}=\sin \left(\frac{\pi}{2} \cup \frac{y_{n}}{m q}\right) .
$$

Herewith, $q=z / n$ denotes the number of slots per pole and phase of the fractional slot stator winding and $y_{n}$ the integer stator winding pitch, respectively. 
Linear magnetic material properties and the usage of a cylindrical structure without any slotting cause Fourier coefficients of the magnetic flux distribution according to

$$
\mathrm{B}_{v} \sim \mathrm{V}_{v}
$$

However, due to the nonlinear material conditions, the varying airgap of the salient poles in addition to the changing slot structure caused by stator and damper slots, we take advantageously use of the assumption

$$
B_{v}=B_{f} \frac{\xi_{v}}{v} g(v)
$$

Now, the magnitudes of the magnetic flux density distribution (6) are not only proportional to the reciprocal value of the harmonic order. B eyond it, there exists a significant influence of the additionally introduced function $g(v)$. The derived relationship (6) allows describing both influences due to the complex geometry and the nonlinear saturation effects in dependence of the harmonic order. However, the fundamental harmonic $B_{f}$ of the flux distribution is always used as a reference value.

In (6), the established polynomial interpolation

$$
g(v)=g_{4} v^{4}-g_{3} v^{3}+g_{2} v^{2}-g_{1} v+g_{0}
$$

has further the ability to describe the numerical obtained nonlinear airgap flux density spectrum $B_{v}$ in dependence of some specific harmonic numbers completely. Thereby, the applied interpolation coefficients $g_{k}, k=0,1,2,3,4$ are varying over a wide range.

Moreover, the very practicable approximation (6) can also be successfully applied to the analysis of the noload, the short circuit as well as the rated operational state. Thus, direct comparisons of the derived harmonic spectrums are further accessible.
The performed investigations are based on a utilized fractional slot stator winding according to Table I with

$$
q=\frac{N}{2 p m}=\frac{z}{n}=\frac{23}{7} .
$$

Thereby, the characteristic of the full pitch winding

$$
\frac{N}{2 p}=9+\frac{6}{7}
$$

gives further rise to take the integer winding pitch

$$
y_{n}=\frac{N}{2 p}-y_{v}=9 \text {. }
$$

Consequently, the utilized fractional slot winding can be characterized with a winding pitch of

$$
\frac{\mathrm{y}_{n}}{\mathrm{mq}}=\frac{63}{69} \text {. }
$$

In the general case of magneto motive force distributions inside the machine, globally statements about both, the possible harmonic numbers $v$ as well as the magneto motive force amplitudes (1) are well known. Especially the undesired effects of disturbing slot harmonics with

$$
v=v^{\prime} \pm 2 k m n q, \quad v^{\prime} \in N_{u}, \quad k \in N
$$

are of crucial interest. The nethermost harmonic number $k=1$ leads in case of $v^{\prime}=1$ in (12) to the lowest slot harmonic orders $v \quad 138 \quad 1$ 


\section{The steady no-load operational state of the synchronous generator}

Two different electrical rotor excitations dealing with the half and the rated rotor field current are discussed in order to acquire a detailed information about the occurring nonlinear saturation effects. The local dependency of the radial magnetic flux density along the circumferential airgap direction is given in Fig.2 over the totally seven poles of the machine in Fig.1. The assigned amplitude spectrum is shown in Fig.3. Thereby, the harmonic space order is related to the seven machine poles.

The derived ratios of each higher harmonic flux density $B_{v}$ to the fundamental magnitude $B_{f}$ with $f=7$ are listed in Table II. Thereby it is obvious, that the effect of iron saturation has the ability to improve the voltage quality due to the significant reduction of higher harmonic magnitudes within higher field excitation currents.

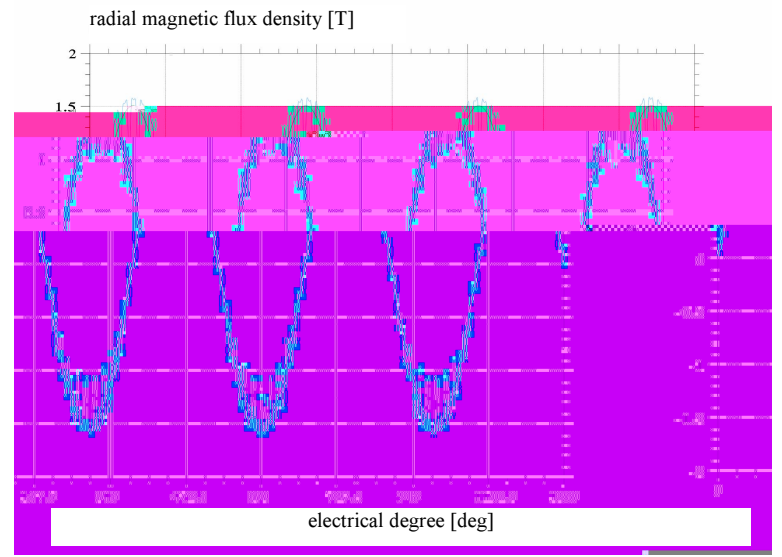

Fig.2. Radial magnetic flux density along the airgap in case of a rotor winding excitation with $\mathrm{i}_{\mathrm{f}}=1.0 \mathrm{pu}$.

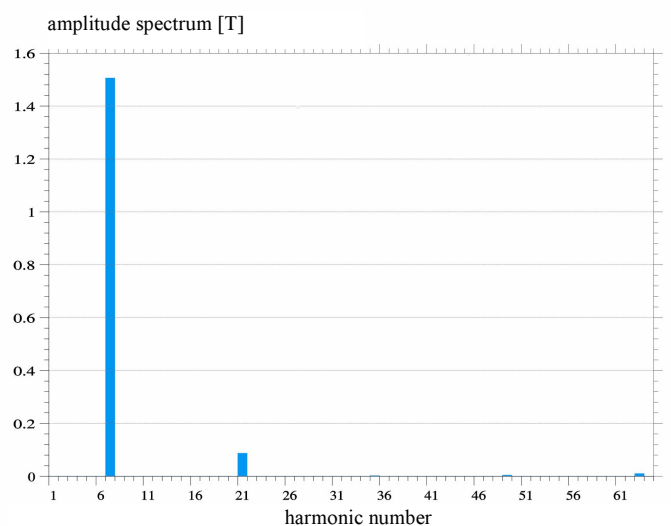

Fig.3. Amplitude spectrum of the radial flux density in case of a rotor winding excitation with $\mathrm{i}_{\mathrm{f}}=1.0 \mathrm{pu}$.

TABLE II. - Amplitude spectrums for various rotor excitations.

\begin{tabular}{cccc}
\hline Harmonic order & Amplitude ratio & $\mathrm{i}_{\mathrm{f}}=0.1 \mathrm{pu}$ & $\mathrm{i}_{\mathrm{f}}=1.0 \mathrm{pu}$ \\
7 & $\mathrm{~B}_{7} / \mathrm{B}_{7}$ & $100 \%$ & $100 \%$ \\
21 & $\mathrm{~B}_{21} / \mathrm{B}_{7}$ & $11.90 \%$ & $5.88 \%$ \\
35 & $\mathrm{~B}_{35} / \mathrm{B}_{7}$ & $3.21 \%$ & $0.20 \%$ \\
49 & $\mathrm{~B}_{49} / \mathrm{B}_{7}$ & $0.48 \%$ & $0.03 \%$ \\
63 & $\mathrm{~B}_{63} / \mathrm{B}_{7}$ & $1.14 \%$ & $0.12 \%$ \\
\hline
\end{tabular}

\section{Magneto motive stator excitation in the magnetic "direct" machine axis}

Two different stator space phasor excitations with low and high magnitudes are applied in the magnetic "direct" axis of the machine depicted in Fig.1. Thus, differences in the harmonic amplitude spectrum of the radial flux density due to primary unknown local saturation effects are obvious.

The rated stator space phasor causes a distribution of the radial magnetic flux density component in the airgap according to Fig.4. The dedicated flux density spectrum is additionally given in Fig. 5 .

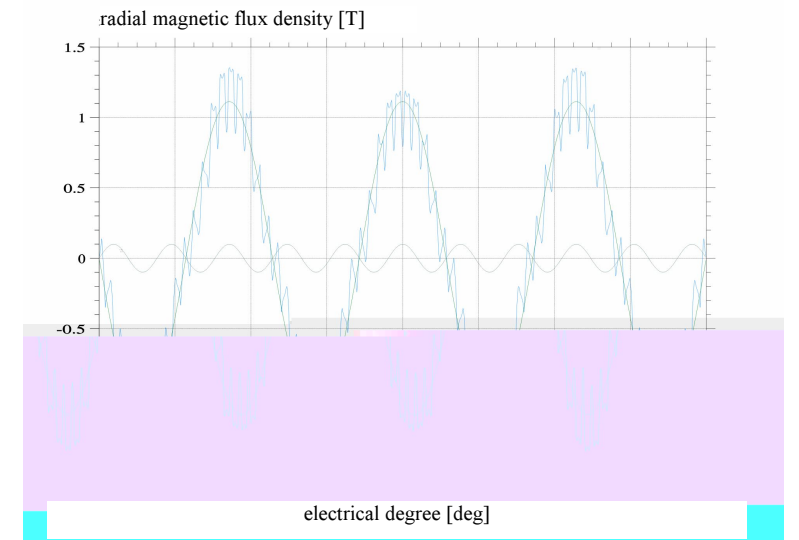

Fig.4. Radial magnetic flux density along the airgap in case of a stator winding excitation with $\mathrm{i}_{\mathrm{s}}=1.0 \mathrm{pu}$ in the "direct" axis.

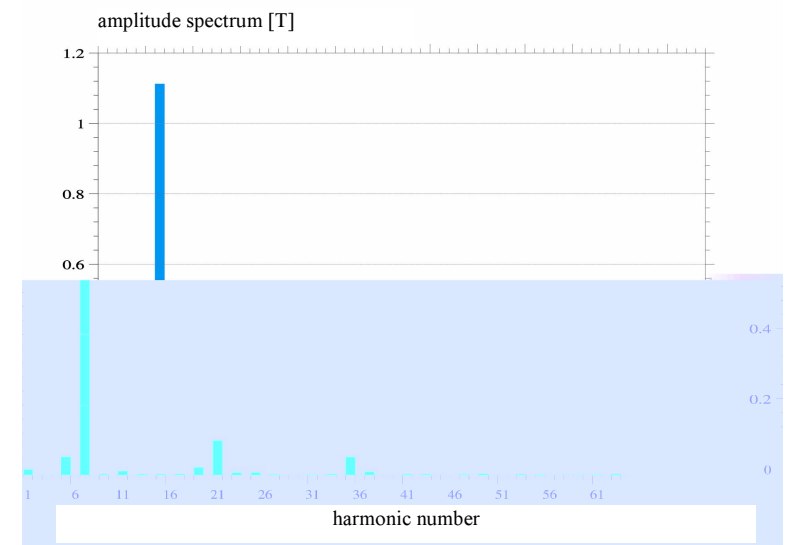

Fig.5. Amplitude spectrum of the radial flux density in case of a stator winding excitation with $\mathrm{i}_{\mathrm{s}}=1.0 \mathrm{pu}$ in the "direct" axis.

TABLE III. - Comparison of harmonics in case of stator phasor excitations in the magnetic "direct" axis.

\begin{tabular}{cccc}
\hline Harmonic order & Amplitude ratio & $\mathrm{i}_{\mathrm{s}}=0.1 \mathrm{pu}$ & $\mathrm{i}_{\mathrm{s}}=1.0 \mathrm{pu}$ \\
1 & $\mathrm{~B}_{1} / \mathrm{B}_{7}$ & $1.95 \%$ & $1.53 \%$ \\
5 & $\mathrm{~B}_{5} / \mathrm{B}_{7}$ & $5.63 \%$ & $4.74 \%$ \\
7 & $\mathrm{~B}_{7} / \mathrm{B}_{7}$ & $100.00 \%$ & $100.00 \%$ \\
9 & $\mathrm{~B}_{9} / \mathrm{B}_{7}$ & $1.13 \%$ & $0.27 \%$ \\
11 & $\mathrm{~B}_{11} / \mathrm{B}_{7}$ & $1.29 \%$ & $1.05 \%$ \\
19 & $\mathrm{~B}_{19} / \mathrm{B}_{7}$ & $2.39 \%$ & $2.01 \%$ \\
21 & $\mathrm{~B}_{21} / \mathrm{B}_{7}$ & $9.91 \%$ & $8.92 \%$ \\
35 & $\mathrm{~B}_{35} / \mathrm{B}_{7}$ & $4.46 \%$ & $4.69 \%$ \\
49 & $\mathrm{~B}_{49} / \mathrm{B}_{7}$ & $0.06 \%$ & $0.04 \%$ \\
63 & $\mathrm{~B}_{63} / \mathrm{B}_{7}$ & $0.03 \%$ & $0.02 \%$ \\
\hline
\end{tabular}


From the comparison of the occurring harmonic ratio $B_{v}$ to the fundamental $B_{7}$, which are listed in Table III for several cases, we deduce that the existing saturation effects due to higher current magnitudes are able to reduce undesired effects for the harmonic orders $v=1,5,9,11,19,21,49,63$. It is in particular remarkable, that the investigated construction leads to a significant magnitude reduction for $v=9$. The only exception is given for the harmonic number $v=35$ whereas a slightly magnitude increasing occurs.

The locally behavior of the most important magnitudes (6) with $v=1,5$ of the complete sub-harmonic spectrum in Table III can alternatively also be approximated by

$$
B_{v} \quad B_{7} \frac{\xi_{v}}{v}\left(\begin{array}{lll}
7.15 v^{2} & 42.4 v & 35.3
\end{array}\right)
$$


The interconnection (6) between the magnitudes of the sub-harmonics with $v=1,5$ in Table IV can be approximated in reference to the fundamental $B_{7}$ according to

$$
B_{v}=B_{7} \frac{\xi_{v}}{v}\left(7.1 v^{2}-41.9 v+34.9\right)
$$

in case of the stator current of $0.1 \mathrm{pu}$ and with

$$
B_{v}=B_{7} \frac{\xi_{v}}{v}\left(7.1 v^{2}-41.9 v+34.8\right)
$$

for the stator current of 1.0pu. Thereby, we can deduced from (17) and (18) that the sub-harmonics $v=1,5$ are not influenced by the magnitude of the stator current. The chosen significant harmonics $v=21,35,49,63$ lead to

$$
\begin{aligned}
B_{v} & =B_{7} \frac{\xi_{v}}{v v}\left(0.0041 v^{4}-0.61 v^{3}+\right. \\
& \left.+29.1^{2}-488+2275\right)
\end{aligned}
$$




\section{The rated operational state}

This operational state can be completely described with distributions of rated stator and rotor space phasors belonging together in different directions. M oreover, they must produce a fundamental magnetic flux density magnitude, which is corresponding to the induced value of the rated voltage.

The according magnetic flux density along the airgap is shown in Fig.10. The obtained amplitude spectrum is obvious from Fig.11, whereby the fundamental harmonic $v=7$ is very distinctive.

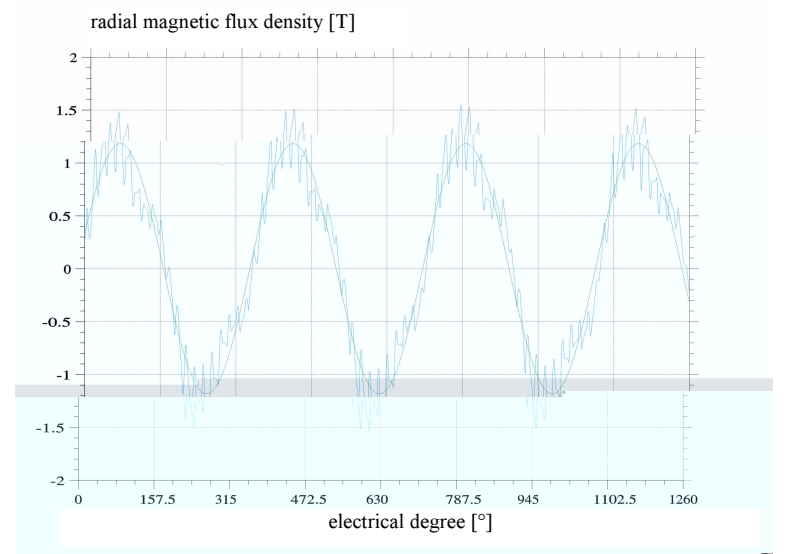

Fig.10. Radial magnetic flux density along the airgap in case of a rotor current of $\mathrm{i}_{\mathrm{f}, \mathrm{N}}=1.0 \mathrm{pu}$, a stator current of $\mathrm{i}_{\mathrm{s}, \mathrm{N}}=1.0 \mathrm{pu}$ and $\mathrm{a}$ phase angle of $\varphi_{\mathrm{N}}=-154.16^{\circ}$.

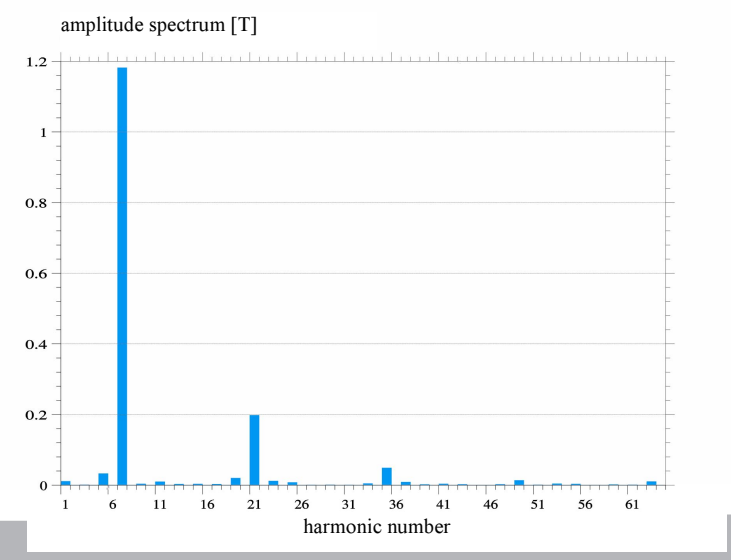

Fig.11. Amplitude spectrum of the radial flux density in case of a rotor current of $\mathrm{i}_{\mathrm{f}, \mathrm{N}}=1.0 \mathrm{pu}$, a stator current of $\mathrm{i}_{\mathrm{s}, \mathrm{N}}=1.0 \mathrm{pu}$ and a phase angle of $\varphi_{\mathrm{N}}=-154.16^{\circ}$.

The dependency of the harmonic magnitudes from the totally harmonic numbers can be summarized in case of the considered sub-harmonics $v=1,5$ with

$$
B_{v}=B_{7} \frac{\xi_{v}}{v}\left(7.38 v^{2}-44.3 v+36.9\right),
$$

whereby the ratios of the interpolation coefficients $g_{2} / g_{1}$ and $g_{2} / g_{0}$ are at least higher than 0.16 . In case of higher harmonics $v=21,35,49,63$ the approximation

$$
\begin{aligned}
B_{v}= & B_{7} \frac{\xi_{v}}{v}\left(0.00013 v^{4}-0.021 v^{3}+\right. \\
& \left.+1.19 v^{2}-28.1 v+234\right)
\end{aligned}
$$

leads for the maximal polynomial order $k=4$ in case of $g_{4} / g_{3}, g_{4} / g_{2}, g_{4} / g_{1}$ and $g_{4} / g_{0}$ due to the term $g_{4} / g_{0}$ to values higher than $5 \cdot 10^{-7}$. The characteristic coefficients $g_{k}$ and the derived ratios are varying in a wide range.

The performed numerical optimization process during the construction period was very successful, in particular for the most important case of the rated operational state. From Fig. 7 it is obvious, that only the desired fundamental and the harmonic component with $v=21$ due to the salient pole structure are significant. All other undesired higher- or sub-harmonics are advantageously vanishing.

\section{Conclusions}

Various typical electrical operational states, such as the no-load, the short circuit and the rated condition, have been investigated and compared with respect to the invoked nonlinear airgap flux density spectrum. M oreover, the sensitivity to local saturation effects has been analyzed in a very detailed way.

Thereby, the influences of the nonlinear iron saturation can also be very useful in e.g. the case of the no-load operational state in order to reduce usually occurring higher harmonics within stronger rotor current excitation.

In the case of an applied stator phasor in the "direct" axis, some higher harmonic magnitudes in the airgap field are decreasing very fast within higher stator current excitations. Contrarily, a stator phasor excitation in the "quadrature" axis shows, that the most harmonics are rising up due to higher stator currents.

During the unlikely event of a short circuit, the salient pole rotor structure causes a very powerful characteristic higher harmonic spectrum in the steady case, whereas the fundamental wave is usually very slow.

A controversial behavior is obvious for the rated operational state of the designed machine, where a distinctive fundamental wave exists in addition to the desired reduced magnitudes of the sub- and higher harmonic components.

\section{References}

[1] A.B.J. Reece and T.W. Preston, Finite Element Methods in Electrical Power Engineering. Oxford University Press Inc., New York, 2000.

[2] K. Ide, M.A. Takahashi, M. Sato, E. Tsuji and H. Nishizawa, "Higher Harmonics Calculation of Synchronous Generators on the Basis of Magnetic Field Analysis Considering Rotor Movement," IEEE Transactions on Magnetics. Vol. 28, No. 2, March 1992.

[3] E. Schmidt, C. Grabner and G. Traxler-Samek, "Finite Element Analysis of the 500 MVA Hydro-Generators at the Bieudron Power Plant," Proceedings of the International Conference on Electrical Machines and Systems, Shenyang (China), 2001. 